\title{
A Study on Effective Strategies for the Intensification of AIDS Education among Chinese College Students
}

\author{
Tao $\mathrm{Wu}^{1, \mathrm{a}}$ \\ ${ }^{1}$ Xi'an Medical University \\ No.1 Xin Wang Road, Weiyang District, Xi'an, Shaanxi, China \\ a18629616661@163.com
}

Keywords: Young students; AIDS prevention and control; Strategy

\begin{abstract}
This With social development, young students in China have become more and more open-minded nowadays. A growing number of them approve of premarital sex, which significantly increases their AIDS infection rate, severely impacting their health and growth. Against this backdrop, this paper discusses the current prevention and control of AIDS infection among Chinese young students, and proposes some strategies to intensify the work.
\end{abstract}

\section{Introduction}

AIDS now has been the second leading cause of death for young adults and teenagers across the globe. From 2014 to 2016, AIDS contributed the most to the death of Chinese young students therefore[1], its prevention and control must not be ignored in China's young adult and teenager health improvement program. At present, some colleges and universities in China are not fully prepared for AIDS prevention and control, giving rise to rapid AIDS epidemics among young students in certain provinces and cities. Additionally, most Chinese young students lack sufficient self-protection awareness, which highly increases their AIDS infection rate[2]. According to relevant studies, 50\% of Chinese young adults and teenagers have sex with their temporary sexual partners, and less than $50 \%$ of them use condoms continuously[3]. Besides, the AIDS infection rate among young males who have sex with men increases on a yearly basis: the rate was $2.9 \%$ from 2005 to 2007 , and it was $6.3 \%$ from 2011 to 2014 , far beyond $5 \%$, the warning line formulated by $\mathrm{WHO}[4]$. There has been high prevalence of AIDS among sexually-active males in China. Nowadays the Chinese government has recognized the importance of educating young adults and teenagers on AIDS prevention, and put forward new requirements and standards for colleges and universities on AIDS prevention.

\section{Causes for AIDS infection of young students in China's colleges and universities}

In China, large educational institutions and health administrative departments have responded positively to the national policies, and have made substantial progress in AIDS prevention and control. However, there still exist many problems in the work of these institutions and departments, and in many areas, AIDS is not fully prevented or controlled. Nowadays, as the situation is increasingly serious, relevant national policies need to be carried out urgently, so as to systematically push and implement the comprehensive strategies to prevent and control AIDS among young students[5].

According to the AIDS epidemic surveillance, the number of young AIDS sufferers in China has risen year by year: in 2008, the number was more than 5000, and in 2015, it was around 10000 . In as short as 7 years, the number doubled[6]. Over $90 \%$ of these young people were infected with AIDS after risky sex behaviors[7]. A report in 2015 showed that the number of young students with AIDS increased by about $35 \%$ compared to that in 2014[8]. On the basis of investigations and studies, the number of young adults and teenagers with AIDS grows much faster than that of other age groups with HIV. The specific reasons are as follows:

\section{Insufficient attention to AIDS prevention.}

Young adults and teenagers are sexually mature, curious about sex and inclined to high-risk sexual behaviors. Although a few of them have gained some understanding of the fact that AIDS can be 
sexually transmitted, they still engage in unsafe sex as their knowledge, attitude and behavior are not always consistent.

In 2016, a survey was carried out in over 60 universities in China. It showed that $43.2 \%$ of the students believed that there was no high prevalence of AIDS, and 56.3\% of them paid no attention to AIDS. The proportion of those who knew elementary knowledge about AIDS was $77.1 \%$, far below $90 \%$, a standard required by China' s State Council. Nowadays $10 \%$ of Chinese young students engage in premarital sex[9]. Among 30000 young students who engaged in premarital sex, less than $50 \%$ of them used condoms in their first sex behavior[3]. These increase the AIDS infection rate among young adults and teenagers drastically.

\section{Underestimation of AIDS infection risks.}

Now Chinese young adults and teenagers are weak in recognizing behaviors that lead to AIDS infection, gravely underestimating their own AIDS infection risks. According to relevant studies, there is a serious lack of awareness and attention to AIDS-related issues among Chinese young adults and teenagers; only $2.8 \%$ of them receive an HIV test. These increase their chance of AIDS infection to a large degree.

\section{Intensification of AIDS prevention and control among young students in China's colleges and universities.}

To establish the outbreak-reporting system as specified.

By establishing the outbreak-reporting system as specified, AIDS prevention and control among young college students can be effectively intensified. In July 2015, the Chinese government dispatched a notice on establishing an outbreak-reporting system to strengthen AIDS prevention and control in educational institutions, specifying that colleges and universities must step up their efforts in this regard[10]. By establishing the outbreak-reporting system, colleges and universities enlarge their reach and are more targeted in the education of AIDS prevention, and young students are served better when they need HIV voluntary counseling and testing as well as behavioral intervention. All of these can enhance AIDS prevention and control in colleges and universities, and effectively curb the AIDS epidemics among young students.

\section{To launch pilot projects to prevent and control AIDS in colleges and universities.}

By launching pilot projects, AIDS prevention and control among young college students can be intensified. There are three main goals for the pilot projects. The first goal is to make sure $100 \%$ of the new students are educated on AIDS prevention by seeking a working mechanism in an effort to enable seminars and health education classes in colleges and universities (a class hour per academic year). The second goal is to make sure over $95 \%$ of the new students are aware of the knowledge on comprehensive prevention and control of AIDSby intensifying the freshmen education and specialized education inside colleges and universities in various ways[11]. The third goal is to seek different sets of the approaches to effective intervention in dangerous behaviors on the basis of practical intervention among key groups of college students. The pilot projects aim to increase the efficiency of AIDS prevention and control, and significantly decrease the infection rate among young students.

\section{To develop credit-sharing courses across colleges and universities.}

To intensify AIDS prevention and control among young students, some courses can be developed whose credits are recognized across colleges and universities[12]. For instance, the course AIDS, Sex and Health can be introduced. It is a course co-developed by Department of Physical, Health and Arts Education of Ministry of Education of China, Disease Prevention and Control Bureau of National Health Commission of China, and zhihuishu.com. It is also the first course on AIDS whose credit can be shared, aiming to enable young students to understand the pathogenesis and route of transmission of AIDS, as well as dangerous behaviors leading to AIDS infection so that they can keep vigilant against AIDS. 


\section{Summary}

In the face of more serious AIDS epidemics among young students in China' s colleges and universities, the outbreak-reporting system should be established as specified, the pilot projects for AIDS prevention and control should be launched, and credit-sharing courses across colleges and universities should be developed. With help of these measures, the young students will understand AIDS better and keep vigilant against it so that their infection rate can be effectively decreased.

\section{Acknowledgements}

Funded by: 2015 Xi' an Medical University Start-up Fund Program for PhDs (NO. 2015DOC26; Name: An Explorative Study on the New Model for AIDS Intervention among College Students) .

\section{References}

[1] Huang Liu. Intensification of AIDS Prevention and Control among Young Students in Colleges and Universities [J]. China Hospital CEO, 2017, (07):62.(in Chinese)

[2] Z. Zhou, etc., An exemplary study on AIDS prevention education in Colleges and universities [J], Chinese University Science \& Technology, 2017(S1):75.(In Chinese)

[3] C. Zhou, etc., Knowledge, beliefs and behaviors of sexually transmitted diseases and HIV / AIDS among college students [J], Foreign Medical Sciences(Section of Medgeography), 2017,38(01):89-91.(In Chinese)

[4] Y. Ma, Challenges and Countermeasures of AIDS prevention education in Colleges and universities [J], Health Medicine Research and Practice, 2015,12(02):5-10.(In Chinese)

[5] L. Yang, etc., Discussion on AIDS prevention and control work in Colleges and universities [J], Health Education and Health Promotion, 2015,10(05):398-400.(In Chinese)

[6] Y. Ma, Adolescents and AIDS [J], Journal of Peking University(Health Sciences), 2016,48(03):385-388.(In Chinese)

[7] X. Chang, etc., Correlation between mental health and coping styles of college students [J], China Journal of Health Psychology, 2016,24(02):277-281.(In Chinese)

[8] Y. Huang, etc., Survey on knowledge about AIDS prevention and treatment among 15 university students in China [J], Health Medicine Research and Practice, 2016,13(04):14-17.(In Chinese)

[9] Yin Ling. AIDS-related Knowledge, Attitude, Behaviors and Intervention Study among Normal College Students [D]. Shandong University, 2017.(In Chinese)

[10] Y. Zhang and L. Han, Study on ethical issues of establishing AIDS notification system in schools [J], Journal of Kunming University of Science and Technology(Social Science Edition), 2016,16(02):18-23.(In Chinese)

[11] Y. Ma, Pushing the Implementation of Comprehensive Strategies for AIDS Prevention and Control among Chinese Young Students [J]. Chinese Journal of School Health, 2017, 38(09):1281-1284.(In Chinese)

[12] Y. Wu, A review of health education on AIDS prevention in Chinese universities in the past 10 years [J], Chinese Journal of School Doctor, 2018,32(05):389-390+393.(In Chinese) 